\title{
POSSIVEIS ETAPAS NA PATOGÊNESE DA CEFALÉIA TENSIONAL E INDICAÇÕES DE TRATAMENTO
}

\author{
CEME FERREIRA JORDY*
}

RESUMO - Análise de resultados obtidos no estudo de uma série de 100 pacientes com diagnóstico de cefaléia tensional permitem sugerir 5 etapas de um processo fisiopatogênico implicado. Com base nesta sugestão, a indicação para o tratamento e respectiva eficácia, dependerão da etapa patogênica sobre a qual se pretenda fazer incidir a ação terapêutica.

PALAVRAS-CHAVE: cefaléia tensional, fisiopatogenia, tratamento, diagnóstico.

Tension headache: possible pathogenic stages and its relations with therapeutic agents

SUMMARY - Five steps in the pathogenic process involved in the tension headache pathogenesis are suggested from a 100 patients clinical study. Therapeutic efficacy in the treatment of the tension headache is considered to be linked to the relation between the therapeutic agent and the stage in which it has focused its effect, in the pathogenic process.

KEY WORDS: tension headache, diagnostic procedures, pathogenetics, strategies of treatment.

A causa imediata da cefaléia tensional está definitivamente comprovada e é de conhecimento geral $^{2,4,6}$. É também conhecido e amplamente demonstrado que nos estados de maior tensão emocional ocorre aumento de níveis de contração muscular ${ }^{1,4,9}$. Como decorrência, não é estranha a comprovação do papel favorável que desempenham medidas que aliviam os estados de tensão emocional, diminuindo ou mesmo interrompendo este tipo de dor. Nosso objetivo neste estudo é analisar mecanismos psicodinâmicos possivelmente envolvidos na gênese da cefaléia tensional, utilizando resultados prévios de avaliação em uma série de 100 pacientes. Naquela ocasião verificou-se aumento da contração muscular (incremento do registro eletromiográfico) desencadeado por estímulo frio (imersão da mão em água a 4 graus Celsius). Entretanto, deixaram de ser analisados então, mecanismos fisiopatológicos que podem estar envolvidos na gênese da cefaléia tensional que nos parecem de importância e justificam voltar ao assunto nesta oportunidade.

\section{MATERIAL E MÉTODD}

São utilizados resultados parciais de estudo realizado em uma série de 100 pacientes, nos quais foi diagnosticada cefaléia tensional. Todos os pacientes haviam já testado sem resultados, outros tipos de tratamento medicamentoso com drogas analgésicas, sedativas e agentes da vasomotricidade. Desse estudo, destacamos o procedimento do estímulo frio que reproduzimos a seguir: cada paciente foi examinado em decúbito dorsal, em cama confortável, à temperatura ambiente de $23^{\circ}$ a $26^{\circ}$ Celsius, com os olhos fechados, em repouso e instruido para se manter quieto, calado, relaxado. A sala de exame é relativamente ampla e silenciosa e com iluminação discreta.

*Livre-Docente, Professor Adjunto de Neurologia da Escola Paulista de Medicina, Professor Colaborador de Neuropsicologia do Instituto de Psicologia da Universidade de São Paulo. Aceite: 20-fevereiro-1995. 
O eletromiograma foi registrado continuamente durante um período de tempo de cerca de 15 minutos, compreendendo 3 fases que se passaram: antes (1), durante (2) e após (3) o estimulo frio. A partir de minutos antes e durante todo o procedimento, a pressão arterial foi registrada manualmente, com garrote no braço, a cada $30 \mathrm{seg}$. $\mathrm{O}$ registros foram analisados visualmente.

Todos os pacientes em que se observou surgimento ou aumento do traçado de interferência na fase 2 e tiveram resposta vasomotora com aumento da pressão arterial máxima até o nível de $20 \mathrm{~mm}$ $\mathrm{Hg}$, foram selecionados para tratamento. Receberam medicaçāo de efeito miorrelaxante e foram revistos para controle do tratamento, 3 a 6 semanas após terem ficado assintomáticos por um período de, no mínimo, 10 vezes superior ao maior intervalo entre as suas crises de dor. Os pacientes que tiveram remissão total e estável dos sintomas foram utilizados para a discussão destes resultados.

\section{RESULTADOS E COMENTÁRIOS}

Em todos os pacientes foi evidenciado: a) presença de atividade muscular na fase1, prévia ao estímulo frio; b) aumento da atividade muscular na fase 2, durante o estímulo frio; c) diminuição da atividade muscular na fase 3 após cessar o estímulo frio mas permaneceu uma atividade residual superior à da primeira fase.

O aumento da tensão muscular provocado por elevação do nível de ansiedade está comprovado. $\mathrm{Na}$ ansiedade aparecem quantidades aumentadas de catecolaminas circulantes que agem diretamente na fibra muscular, provocando contração. Outros mecanismos de ação central dependentes da liberação de catecolaminas e substâncias simpaticomiméticas estão envolvidos no desencadeamento da agitação psicomotora e tremores generalizados que fazem parte da crise de ansiedade. Nos pacientes desta série a atividade muscular, presente em todas as fases do eletromiograma, pode ser interpretada como sinal de aumento da tensão muscular consequente a níveis elevados de ansiedade ${ }^{3,4,8}$. Além disto, durante o exame a brusca elevação da ansiedade provocada pelo agente frio stressor causou, nos pacientes, aumento crítico da contração muscular eletromiograficamente demonstrado na cabeça e pescoço. Este mecanismo, entretanto, nem sempre opera. Nāo está presente, por exemplo, na parte da população dos pacientes examinados que, não tendo apresentado reação muscular ao estímulo frio, não foram selecionados para esta série.

O resultado positivo da prova terapêutica confïrma a existência da contração muscular na gênese da dor, desde que todos os pacientes receberam medicamentos de efeito miorrelaxante, tendo procurado novo atendimento médico, em vista do fracasso de tratamentos previamente feitos com analgésicos, sedativos ou drogas com ação sobre a vasomotricidade. A contração muscular parece ter sido a causa imediata da dor, como tem sido verificado em outras séries ${ }^{2,379}$. Se a ansiedade provoca aumento da contração muscular e esta é comprovadamente a causa da dor na cefaléia tensional, cria-se uma sequência de eventos que conduzem ao aparecimento da cefaléia, por aumento da ansiedade e o surgimento de contração muscular excessiva na cabeça.

A ansiedade é um processo, cuja intensidade varia na dependência de fatores originados no meio ambiente e no mundo interno ${ }^{4,5}$. É uma síndrome bem conhecida quanto aos aspectos semiológicos, tanto em suas manifestaçōes críticas - a crise de ansiedade ou crise de pânico - quanto em suas manifestaçōes crônicas ${ }^{1.4}$. Enquanto as primeiras se apresentam em exuberante constelação aberta de sintomas e sinais requerendo sempre atendimento urgente, a ansiedade crônica pode ficar contida em vagas percepções, que o paciente com frequência deixa de identificar como algo anormal considerando-as, em geral, 'parte integrante e natural da sua personalidade' e assim não merecendo, a seu ver, qualquer cuidado terapêutico. Pode-se admitir porém, que a ansiedade crônica soma, a cada nova influência, um já permanente componente favorecedor patogênico que existe como um 'nivel constante de ansiedade'. Desta forma, tanto maior será a ansiedade em dada situação diante de um agente stressor, quanto maior for o nivel permanente crônico de ansiedade, vivido pelo paciente. Sob influência de fatores internos ou externos, em razão de vivências experimentadas pelos pacientes 
na relação com seu universo formam-se flutuações no estado de ansiedade podendo, em momentos ou períodos da vida, fazer surgir o 'sintoma' gerado por necessidades impostas do aparelho psíquico e que têm origem em conteúdos e mecanismos intrapsíquicos inconscientes. Construido sob paradigmas do aparelho psíquico o sintoma é, sob o prisma psicológico, uma síndrome ininteligivel senāo com os recursos que entendem a dinâmica intrapsíquica.

As características clínicas das crises de dor, seus fatores desencadeantes imediatos, o comportamento dos pacientes durante as crises, os resultados do interrogatório complementar sobre aspectos de personalidade e, especialmente a resposta muscular ao aumento brusco da ansiedade verificados nesta série, fazem-nos pensar que a contração muscular e demais fenômenos que compõem a síndrome da cefaléia tensional podem ser interpretados como um sintoma sob o prisma psicodinâmico e originado, portanto, nos mecanismos intrapsiquicos inconscientes expostos. A eles deve ser dirigida a atenção em busca da compreensão do processo e da estratégia terapêutica. Segundo este critério, no encadeamento de fenômenos que compõem o mecanismo patogênico da cefaléia tensional podem ser identificadas as seguintes etapas: a) núcleos e mecanismos patogênicos intrapsíquicos inconscientes b) ansiedade crônica c) fatores ansiogênicos (stressores) ambientais ou internos; d) contração dos músculos da cabeça e pescoço; e) dor. Da consideração desta cadeia de fenômenos deve surgir a compreensão dos recursos terapêuticos que norteiem o tratamento da cefaléia tensional.

As considerações apresentadas levam-nos a admitir que a ação terapêutica dirigida ao processo da cefaléia tensional será tão mais eficaz, quanto mais se acercar da etapa patogênica primordial. Todas as medidas terapêuticas situam-se, portanto, em um 'continuum crescente' quanto à sua eficácia, à medida que se distanciem da fase final da cadeia patogênica, a dor, e se aproximem da etapa inicial, o provável núcleo original inconsciente da síndrome da cefaléia tensional.

Agradecimento - Agradeço à Sra. Ivany S.Jordy, pelas leituras e criticas e o incentivo que contribuiram para a elaboraçāo deste estudo.

\section{REFERÊNCIAS}

1. Borgeat $F$, Hade B, Elie R, Larouche LM. Effects of voluntary muscle tension increases in tension headache. Headache 1984, 24:199-202.

2. Classification and Diagnostic Criteria for Headache Disorders, Cranial Neuralgias and Facial Pain. Cephalalgia, 1988, 8 (Suppl 7).

3. Cohen MJ, McAarthur DL. Classification of migraine and tension headache from a survey of 10000 headache diaries. Headache, 1981, 21:25-29.

4. Haber JD, Kuczmierczyk AJ, Adams HE. Tension headaches: muscle overactivity or psychogenic pain. Headache 1985, 25: 23-29.

5. Holm JE, Holroyd KA, Hursey KG, Penzien DB. The role of stress in recumrent tension headache. Headache 1986, 26:160-167.

6. Parckard RC. What is psychogenic headache?. Headache 1976, 16:20-23.

7. Sturgis ET, Schaefer CA, Ahles TA, Sikora TL. Effect of movement and position in the evaluation of tension headache and nonheadache control subjects. Headache 1984, 24:88-93.

8. Weeks R, Baskin S, Rapoport A, Sheftell F, Arrowsmith F. A comparison of MMPI personality data and frontalis electromyographic readings in migraine and combination headache patients. Headache 1983, 23:75-82.

9. Ziegler DK. Headache syndromes: problems of definition. Psychosomatics 1979, 20:443-447. 